\title{
EPA Forum 2019
}

\section{EPA Forum 2019: Implementing Comprehensive, Evidence-Based and Rights-Centred Strategies for Mental Health Care across Europe}

\section{EPAF-0001}

\section{ROAMER and Horizon 2020: priorities in clinical mental health research \\ J.M. Haro}

Parc Sanitari Sant Joan de Deu- CIBERSAM, Teaching and Research

Unit, Sant Boi de Llobregat- Barcelona, Spain

Mental disorders most likely represent the greatest health burden to Europe - not only for directly affected individuals, but also for caregivers and wider society. However, the investment in research to face this challenge is largely below the size of the problem. The ROAMER project, funded by the European Commission FP7 program, established a comprehensive, coordinated mental health research agenda for Europe and the world that might reduce the costs and burdens of mental disorders. This agenda was integrated and revised through surveys and consensus decision-making by more than 1000 scientists and affected stakeholders including individuals with mental health problems and their families, healthcare workers as well as policymakers and funders to produce 6 high-level priorities for mental health research. The project finished about 3 years ago and aimed to having an impact on the research priorities in Europe. With FP8 research program (Horizon 2020) coming to an end this paper will review the investment in mental health research in Europe in the last years. This revision is hampered by the diversity of funding instruments and the lack of an overall strategy in mental health research by the European Commission and the member states. Now that a new Framework Program (FP9, Horizon Europe) is being designed, this discussion may provide some areas of interest to be included in it.

Disclosure of interest.- The authors have not supplied a conflict of interest statement.
EPA Forum.- EPA Forum 2019: Implementing Comprehensive, Evidence-Based and Rights-Centred Strategies for Mental Health Care across Europe.

\section{EPAF-0002}

\section{The users' and EPA perspective}

A. Nomidou GAMIAN general secretary, Belgium

Society has traditionally viewed persons with mental disabilities as objects of charity and social welfare, and laws have historically considered them as being incompetent, dangerous and violent and emphasized protection. The human rights of people with mental disabilities have frequently been disregarded or devalued within the healthcare system, sometimes with the best of intentions. The UNCRPD is the first legally binding instrument with comprehensive rights and protections for persons with mental disabilities on an equal basis with others. Stereotypes leveled against persons with mental health diagnoses are impermissible after States have acceded to the UNCRPD and over-reliance on the confining logic of a purely biomedical model displays an imperfect understanding of the UNCRPD. However, twelve years on from the adoption of the UNCRPD and there is still a wide gap between the human rights legal frameworks and aspirations and the reality for people with mental disabilities. Legal and policy approaches are not enough. A shift in the conceptualization of healthcare for people for mental disabilities through a human rights lens is a key element and changes will be required at multiple levels, including knowledge, attitudes and practices. More collective dialogues among health professionals and persons with mental disabilities are needed to reach common goa ls in a reciprocal way. The European Psychiatric Association and the patient-driven Global Alliance of Mental Illness Advocacy Networks-Europe moved towards this direction and signed a Memorandum of Understanding on Collaboration in their respective fields of mutual interest.

Disclosure of interest.- The authors have not supplied a conflict of interest statement. 